\title{
LOS ESPACIOS DE TUTORÍA EN PRÁCTICA PROFESIONAL Y SUS NECESIDADES DE FORTALECIMIENTO \\ TUTORING SPACES AND PRACTICE BUILDING NEEDS
}

\author{
Volumen 14, Número 2 \\ Mayo - Agosto \\ pp. 1-25
}

Este número se publicó el 30 de mayo de 2014

Gerardo Ignacio Sánchez Sánchez

Ximena Elizabeth Jara Amigo

Revista indizada en REDALYC,

Revista distribuida en las bases de datos:

CATÁLOGO DE LATINDEX, IRESIE, CLASE, DIALNET, DOAJ, E-REVIST@S, SHERPA/ROMEO, QUALIS, MIAR

Revista registrada en los directorios:

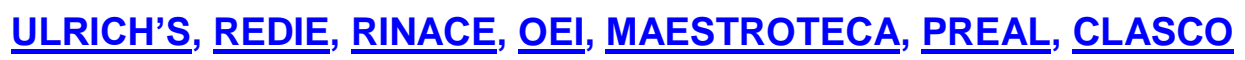




\title{
LOS ESPACIOS DE TUTORÍA EN PRÁCTICA PROFESIONAL Y SUS NECESIDADES DE FORTALECIMIENTO TUTORING SPACES AND PRACTICE BUILDING NEEDS
}

\author{
Gerardo Ignacio Sánchez Sánchez ${ }^{1}$ \\ Ximena Elizabeth Jara Amigo²
}

\begin{abstract}
Resumen: El artículo da cuenta del funcionamiento de la tutoría en práctica profesional, según el reporte de los actores que viven el proceso: estudiantes de pedagogía en Educación Física, General Básica, Parvularia, Historia, Castellano, Matemática, Artes e Inglés de una institución formadora de profesores en la región del Maule, Chile y sus tutores. Participaron 225 estudiantes en práctica y 57 tutores universitarios; metodológicamente asume la modalidad de estudio descriptivo de caso múltiple. Los resultados indican opiniones bastante positivas respecto al sistema de acompañamiento diseñado por la universidad para los estudiantes en práctica profesional y que se concretan con la presencia de un profesional encargado de apoyar las exigencias personales, sociales y profesionales que experimentan los practicantes. Existe una alta valoración de la tutoría en tareas de apoyo a la planificación, la didáctica, la evaluación y la reflexión. Sin embargo, la visión que existe de los desempeños logrados tiende a ser distinta entre los actores. Los practicantes perciben un desempeño que, en términos generales, se sitúa en los niveles de "bastante" y "mucho", lo cual indicaría que ellos consideran adquiridos los distintos desempeños pedagógicos; en tanto, los tutores sitúan a un porcentaje importante de practicantes en una adquisición más bien parcial o "regular" de los desempeños pedagógicos comprometidos en la formación de un profesor.
\end{abstract}

Palabras clave: PEDAGOGIA, FORMACIÓN DOCENTE, PRÁCTICA PROFESIONAL, TUTORÍA, CHILE

\begin{abstract}
The article is a report of the actors that have lived the process of practicum and their tutoring sessions. This involves students of pedagogy in physical education, basic general, preschool, history, Spanish, mathematics, arts and English, in a teacher training institution in the region of Maule, Chile and their tutors. 225 students in their practicum process and 57 college tutors participated in this work. The research was in the form of descriptive multiple case study. The results indicate very positive opinions regarding the support system, designed by the University for the students in professional teaching practice and which are specified in the presence of a professional responsibility to support the demands personal, social and professional experienced practitioners. On the other hand, it emphasizes that while recognizing mentoring as a support space classroom practitioner performance with regard to planning, teaching, assessment and reflection, the vision of the performances achieved there tends to be different among the actors. Practitioners perceive a performance that, in general, is at the level of "sufficiently" and "a lot", which would indicate that they consider acquired the different performances pedagogical acquired; however, the tutors' view is different, and place a significant percentage of practitioners in an acquisition rather partial or "regular" of educational performances engaged in the formation of a teacher.
\end{abstract}

Key words: PEDAGOGY, TEACHER TRAINING, PROFESSIONAL PRACTICE, TUTORING, CHILE.

\footnotetext{
${ }^{1}$ Actualmente, se desempeña como académico de la Facultad de Educación de la Universidad Autónoma de Chile. Profesor de Historia y Geografía. Magíster en Política y Gestión Educacional por la Universidad Talca de Chile. Doctor en Ciencias de la Educación por la Universidad de Serena. Dirección electrónica: gsanchezs@uautonoma.cl/gerignsan@gmail.com

${ }^{2}$ Actualmente se desempeña como Secretaria de Estudios de la carrera de Historia, Geografía y Ciencias Sociales de la Universidad Autónoma de Chile. Profesor de Historia y Geografía. Magister en Curriculum y evaluación por la Universidad Arcis de Chile. Estudiante de Doctorado en Cultura y Educación por la Universidad Arcis. Dirección electrónica: xjaraa@uautonoma.cl
}

Artículo recibido: 26 de octubre, 2013

Devuelto para corrección: 17 de diciembre, 2013

Aprobado: 15 de mayo, 2014 


\section{Introducción}

Los requerimientos actuales de la docencia se orientan a contar con profesionales de alto nivel en el área de educación, con autonomía intelectual y capacidad de reflexión, que le permitan aportar a las disciplinas de manera significativa, favoreciendo el aprendizaje de sus estudiantes. En ese contexto, los modelos actuales de formación inicial de profesores otorgan una función central a las experiencias de inserción en centros educacionales. La formación práctica ofrece a los futuros profesores oportunidades para asumir un rol protagónico y, desde allí, comprender y desarrollar las competencias involucradas en el ejercicio profesional (Montecinos, Solís, Contreras y Rittershaussen, 2009, p. 47).

La práctica profesional, como última etapa de formación en la malla curricular de los estudiantes de pedagogía, representa un hito de gran importancia para los futuros profesores, lo que no está exento de incertidumbre e interrogantes respecto de lo que significa ser un buen maestro y cómo realizar una práctica exitosa (Galaz, 2011, p. 48).

A pesar de los esfuerzos por articular contenidos, desarrollar competencias y vincular gradualmente a los futuros profesores con el medio laboral en que se desempeñarán, la presión del "buen desempeño" que el entorno les impone provoca en los estudiantes altos niveles de incertidumbre y temores, despertando múltiples interrogantes sobre si poseen las competencias requeridas en un buen profesor (Galaz, 2011, p. 49).

En ese escenario, la Facultad de Educación de la Universidad Autónoma de Chile, sede Talca, ha definido un modelo de formación que entiende la práctica como una acción profesional que presupone que, en la base de los sujetos en formación, existe alguna idea sobre la enseñanza en materia de métodos, junto con nociones acerca de la procedencia del conocimiento. Esta acción se encuentra sostenida en un cuerpo teórico de conocimientos y requiere de un periodo de formación académica. Proporciona un saber que permite encaminar a los estudiantes hacia la deliberación para intervenir profesionalmente en la compleja vida del aula y de la escuela.

La definición de esta línea de formación como profesional trasciende la adquisición de habilidades y destrezas o competencias prácticas, supone adquirir valores, intereses y actitudes que caracterizan la profesión y, por lo tanto, asumirla como eje de la formación docente. En tal caso, los futuros docentes deben disponer del conocimiento necesario para enseñar y poseer competencias y capacidades, analizar su práctica y estar consciente de las implicaciones ético-valorativas de la enseñanza. 
Anclada en cuatro ejes de acción: (1) el conocimiento pedagógico del contenido; (2) prácticas o experiencias prácticas; (3) efectividad en la diversidad y (4) reflexividadinvestigación y articulada en una línea de formación progresiva, genera las condiciones para estimular la producción de conocimiento, la reflexión y acción integradora en un trabajo teórico-práctico, relacionados con procesos pedagógicos desde distintas intencionalidades formativas. Se concibe la formación práctica como un espacio o itinerario de trabajo orientado a promover el cuestionamiento de la teoría a partir del enfrentamiento con problemas educativos y la búsqueda de posibles soluciones; conforme al perfil del educador propuesto.

Los programas de Pre-Práctica y Práctica profesional brindan al estudiante la oportunidad de fortalecer los conocimientos adquiridos en el proceso de formación, desempeñándose como docente responsable en el curso o subsector que le corresponda, incorporándose como miembro activo partícipe en todas las actividades propias del establecimiento.

Desde el punto de vista de sus objetivos, se espera que el profesional evidencie un desempeño que le permita (a) cumplir con la función de mediador de conocimiento, actitudes y valores que se esperan sean asumidos como propios por sus alumnos, relacionando estas funciones con la tarea de enseñar; (b) asumir su condición de aprendices permanentes y adoptar una práctica constante de análisis crítico respecto de su trabajo docente, contrastando siempre sus expectativas con la realidad y (c) reflexionar antes y después de enseñar como una estrategia de educar y de retroalimentación permanente de sus prácticas pedagógicas (Universidad Autónoma de Chile, 2012)

Para apoyar dicho proceso, la universidad contempla la participación de un equipo contratado de tutores de práctica, con título en la especialidad y experiencia en el ejercicio de la docencia. Sus tareas se orientan a: (a) desarrollar un trabajo colaborativo con los diferentes integrantes del equipo de prácticas, respetar las decisiones acordadas con la coordinación de prácticas y asistir a las reuniones citadas por dicho organismo; (b) mantener una comunicación asertiva que permita las buenas relaciones entre instituciones y el buen desempeño del proceso de prácticas de los estudiantes en relación con los directivos del centro educativo; (c) orientar a los profesores guías de establecimientos acerca de las funciones que asumirán, plantear con ellos el trabajo de orientación y supervisión de los practicantes; (d) realizar sesiones semanales de información, organización, acompañamiento y evaluación con los profesionales en formación en el trascurso de la práctica. 
Profundizar en este escenario, estudiando las opiniones de los propios estudiantes y tutores al respecto, validando así su experiencia en las instancias de práctica y lo que ellos mismos han relevado como características de un buen acompañamiento, tiene como propósito ofrecer información útil a los formadores de profesores respecto a cómo mejorar y estructurar espacios e interacciones que provean el andamiaje, más que la resistencia, para el desarrollo de un adecuado desempeño profesional de los futuros profesores. Particularmente, cuando los programas de formación inicial de docentes en Chile, así como en otras partes del mundo, requieren, cada vez más, entregar evidencias respecto de su calidad y efectividad (Montecinos et al., 2009, p. 15).

\section{Breve Referente Teórico}

La formación docente en Chile es de larga data. En el último tiempo, la calidad de la formación de los profesores ha sido una fuente de preocupación y de interés para el Estado y la Sociedad en su conjunto. Así lo evidencia, al menos, la puesta en marcha de diversos dispositivos tales como el Programa Inicia, la definición de Estándares de Egreso, los Programas de Mejoramiento de la Calidad de la Educación Superior (MECE- SUP), entre otros.

Sin embargo, un velo de sombra parece rodear los procesos de formación inicial en tanto no logran responder a las exigencias actuales impuestas por la sociedad, en términos de asegurar, en los egresados, un conjunto de competencias que los habiliten para el ejercicio profesional y para incidir favorablemente en los niveles de calidad y equidad que rodean al sistema.

En un contexto de abierta masificación, la formación inicial se ve enfrentada, por una parte, a estudiantes con heterogeneidad de capitales culturales y por otra parte, a crecientes demandas en términos de asegurar el desarrollo de un conjunto de estándares que están en la base de lo esperable de la gestión de un profesor.

La problemática antes descrita pone de manifiesto la necesidad de develar el tipo de conocimiento que se configura en la formación inicial de docentes, los contextos donde se desarrolla de mejor manera el proceso, así como los actores involucrados en dicho proceso, dado el imperativo de comprender mejor la racionalidad subyacente en las distintas propuestas de formación de docentes. 
En la realidad se mantiene una lógica dicotómica, donde cada uno de los componentes mencionados termina siendo leído desde la preponderancia de uno sobre otro. Sin embargo, la evidencia empírica sostiene que es posible, aproximarse a una forma distinta de concebir el proceso formativo, donde las oportunidades de práctica que posee el futuro docente se constituyen en el espacio de síntesis de cada uno de los componentes descritos previamente.

Entonces, el problema no es por el tipo de conocimiento predominante (teórico-práctico) en un determinado contexto (universidad-escuela) con un actor protagónico (académicoestudiante en práctica-docente del centro escolar), sino la constitución de un espacio formativo donde dichos componentes en su conjunto favorezcan el proceso formativo del futuro docente (Martin, Snow y Franklin, 2011) y en el que se ponen a prueba.

En ese contexto, el proceso de formación docente ha sido objeto de múltiples investigaciones en las cuales se ha observado "una gran insatisfacción tanto de las instancias políticas como del profesorado en ejercicio o de los propios formadores respecto a la capacidad de las actuales instituciones de formación para dar respuesta a las necesidades de la profesión docente" (Marcelo, 2008, p. 10).

Dentro del descontento que existe en la formación inicial del profesorado, se hace latente el divorcio entre la teoría y la práctica, es decir, la hegemonía de los elementos teóricos frente a los elementos prácticos (Marcelo, 2008, p.157). Además, existiría evidencia de que las experiencias prácticas tempranas fortalecen la vocación docente, la motivación por la carrera y el compromiso con su propia formación profesional.

En ese sentido, Macelo, citando a Pérez, menciona que "la práctica se concibe como el espacio curricular especialmente diseñado para aprender a construir el pensamiento práctico del profesor en todas sus dimensiones" (1988, p. 31). Por consiguiente, y citando a Ball y Cohen, destacan tres condiciones necesarias si se espera algún aprendizaje a partir de la experiencia práctica:

a) Los profesores tienen que aprender a adecuar sus conocimientos a cada situación, eso significa indagar acerca de lo que los estudiantes hacen, piensan y cómo comprenden lo que se les ha enseñado.

b) Los profesores deben aprender a utilizar su conocimiento para mejorar su práctica.

c) Los profesores necesitan aprender cómo enmarcar, guiar y revisar las tareas de los alumnos desde su punto de vista, la clave de nuestra respuesta es que estar centrado 
en la práctica no necesariamente implica situaciones en las aulas en tiempo real (2008, p. 23).

Lo que sí está claro es que la práctica constituye un espacio dentro del itinerario formativo caracterizado como un momento de particular complejidad entre la alegre teoría en las nubes y la dura práctica cotidiana. En ese sentido, numerosos especialistas a la hora de justificar su práctica tienden a referirse "a los modelos teóricos basados en la filosofía que profesan, que pueden o no coincidir con su teoría al uso. Entendiéndose como "teoría profesada", lo que los docentes dicen que hacen en una situación dada, en la que en verdad creen, y la "teoría al uso", es decir, lo que ellos en realidad hacen (Brockbank y McGill, 2002, p. 43). Finalmente, es en la praxis, donde se puede evidenciar si la teoría profesada por los profesionales coincide con la teoría en uso.

Enfrentar los desafíos de la docencia requiere que los futuros docentes sean capaces de adquirir el compromiso de la "práctica reflexiva", tal como lo señala Brockbank y McGill (2002, p.46), porque dicha práctica "da oportunidad a los profesores para examinar sus filosofías subyacentes, evaluar su utilidad en el contexto de la enseñanza y considerar enfoques alternativos basados en otras filosofías o combinaciones de estas".

Avanzar en el desafío de hacer realidad el desarrollo de la habilidad reflexiva supone la definición de un sistema de acompañamiento, con profesores tutores y guías capaces de generar un ambiente mediado de interacciones que permitan a los futuros docentes aprender a pensar, a hablar y actuar como profesores

En ese sentido, Álvarez (2002, p. 34) afirma que los estudiantes en práctica han de ser orientados en este proceso a través de profesores tutores "quienes compaginan y globalizan de forma estructurada en el proceso formativo aquellos conocimientos que se consideran relevantes y significativos para el estudiante tutorizado". En un desafío formativo que se orienta a favorecer la autorregulación en el aprendizaje, la madurez vocacional y la capacidad de adaptación a un mundo en permanente transformación. Y que les presenta los problemas de la profesión de forma integrada, no en compartimentos estancos.

En consecuencia, la tutoría es definida por Álvarez (2002, p. 34) como un proceso cooperativo de acciones formativas y secuenciadas estrechamente vinculadas a la práctica educativa y con una clara proyección hacia la madurez global del individuo, mediante las 
cuales se enseña a aprender, comprender, reflexionar y decidir de manera comprometida, responsable y autónoma.

En ese contexto, existirían habilidades y conocimientos específicos que debiera poseer un buen tutor con el fin de ayudar a los estudiantes en su proceso de formación. Malderez (2004, p. 19) plantea tres facetas de la "reflexión", que resultan fundamentales en la labor de un tutor: holding up the mirror, thinking over y modelling.

Queda claro que el propósito del profesor tutor es demostrar los principios de enseñanza-aprendizaje al estudiante en práctica, estableciendo la tutoría como una relación de ayuda que va más allá de la resolución de problemas puntuales y concretos, para ampliarse a la dinámica que conforma el proceso formativo en toda su extensión.

La importancia del profesor tutor radica en ayudar a los estudiantes en práctica a valorar su situación personal ante el mundo que les rodea y a adoptar una actitud crítica y transformadora para encontrar salida a los problemas que a diario ha de resolver cada uno. Para llevar a cabo esta labor de ayuda, la intervención del profesor tutor no puede desarrollarse al margen del entorno social en que está inmerso el practicante, sino en estrecha vinculación de éste.

Por tanto, se puede decir que el tutor es un profesional de la enseñanza que ayuda al estudiante en su recorrido por el sistema educativo "formando actitudes favorables hacia el conocimiento, hacia el estudio, facilita el mapa para que la ruta se recorra de manera adecuada, retira algunas piedras del camino que dificultan dicho trayecto, fomenta la creatividad, la autonomía, la responsabilidad, etc." (Álvarez, 2002, p. 47) Es, entonces, un formador que involucra al estudiante en su propio aprendizaje, abordando distintos aspectos del proceso formativo y madurativo, que no están recogidos en el programa de cada materia, ni en el plan de estudios.

Por consiguiente, Malderez (2004) señala que existen cinco roles y funciones principales que ha de tener un tutor: (1) modelo (inspirar y demostrar), (2) aculturador (explicar al practicante cómo realizar una actividad; ayudar a comprender y hacer uso de una cultura profesional en particular), (3) auspiciador (abrir puertas, integrar a los estudiantes en práctica con las personas apropiadas, usar su poder -la habilidad de hacer que las cosas pasen- en servicio de los estudiantes en práctica), (4) apoyo (estar presente, proveer de oportunidades para que los alumnos se expresen y dejen fluir sus emociones y actuar como una caja de resonancia ante situaciones inesperadas) y (5) educador (actuar como un referente para 
articular ideas, crear conscientemente las oportunidades apropiadas de aprendizaje para el estudiante en práctica y a alcanzar objetivos de aprendizaje).

\section{Metodología}

El estudio que pretende dar cuenta de la visión de estudiantes y tutores respecto al funcionamiento de los espacios de tutoría en práctica profesional, desde el punto de vista metodológico, se desarrolló bajo un enfoque de carácter cuantitativo, entendido como un conjunto de procesos secuenciales y probatorios. Se inscribe dentro de los estudios descriptivos, puesto que se busca evidenciar el desarrollo de los espacios de tutoría en práctica profesional, especificando las orientaciones que entregan los profesores tutores a estudiantes en práctica profesional de las carreras de pedagogía. Todo esto con el propósito de detallar cómo se manifiesta el fenómeno de interés sometido a análisis.

El diseño de investigación admite un carácter "no experimental-transeccional", pues es un estudio que se realiza deliberadamente, es decir, se trata de una investigación, donde no se modifican, en forma intencional, las variables. Lo que hace la investigación no experimental es observar fenómenos, tal y como se dan en su contexto natural para después describirlo. Este se realiza en un solo momento, en un tiempo único. Desde el punto de vista de su modalidad, se inscribe en los estudios de casos múltiples.

La población en estudio estuvo representada por las ocho carreras de pedagogía, pertenecientes al último año de formación universitaria. La unidad de análisis está configurada por:

- Profesores Tutores: docentes contratados por la Universidad Autónoma de Chile con el fin de supervisar y tutorizar a los alumnos en su proceso de práctica profesional. Participaron 57 tutores de práctica.

- Estudiantes en práctica: estudiantes que cursan último año de las carreras de pedagogía. Participaron 225 estudiantes en práctica profesional.

A continuación se presenta en la Tabla 1, la distribución por carrera, del número de estudiantes y tutore que participaron en el estudio. 
Tabla 1

Distribución de participantes

\begin{tabular}{|l|l|l|}
\hline \multirow{2}{*}{ Carrera } & \multicolumn{2}{|c|}{ TALCA } \\
\cline { 2 - 3 } Castellano & Estudiantes & Tutores \\
\hline Artes visuales & 26 & 10 \\
\hline Básica & 5 & 5 \\
\hline Educación Física & 39 & 9 \\
\hline Historia & 15 & 4 \\
\hline Inglés & 33 & 4 \\
\hline Matemática & 48 & 9 \\
\hline Parvularia & 18 & 4 \\
\hline Total & 41 & 12 \\
\hline
\end{tabular}

Fuente: elaboración propia del autor (2012)

En este trabajo se dispuso de un cuestionario aplicado a los profesores tutores y a los estudiantes en práctica profesional en línea, con el fin de describir las opiniones respecto al funcionamiento del espacio de tutoría, específicamente en cuanto a: (a) diseño del plan de clases del estudiante, (b) plan-acción de tutorías y (c) desempeños pedagógicos evidenciados en los escenarios de práctica.

Estos cuestionarios están diseñados bajo el formato de escala de apreciación, pues cada una de las afirmaciones va acompañada de una escala que permite dar cuenta del nivel de adquisición, pudiéndose puntuar del siguiente modo: (1) No posee en absoluto, la desconoce; (2) La posee poco; (3) La posee medianamente; (4) La posee bastante; (5) La posee totalmente, la domina.

Los datos obtenidos de dichos cuestionarios fueron analizados a partir de las dimensiones definidas en los objetivos de estudio, haciendo uso de SPSS.

\section{Resultados y análisis}

\section{Estudiantes en Práctica}

Se presenta en la tabla 2, la visión de los estudiantes en práctica respecto al funcionamiento de la tutoría, en el ámbito del diseño del plan de clases. 
Tabla 2

Ámbito: Diseño del Plan de Clases

\begin{tabular}{|l|c|c|c|c|c|}
\hline Ámbitos Diseño del plan de clases & 5 & 4 & 3 & 2 & 1 \\
\cline { 2 - 6 } Mi tutor de práctica... & (Mucho) & (Bastante) & (Regular) & (Poco) & (Nada) \\
\hline $\begin{array}{l}\text { Retroalimenta las planificaciones } \\
\text { clase a clase presentadas. }\end{array}$ & 101 & 78 & 30 & 10 & 6 \\
\hline $\begin{array}{l}\text { En relación a la comprensión del } \\
\text { contenido a enseñar, me ayuda a } \\
\text { establecer vinculaciones con los } \\
\text { objetivos transversales. }\end{array}$ & 106 & 81 & 26 & 9 & 3 \\
\hline $\begin{array}{l}\text { Me sugiere estrategias diversas para } \\
\text { la presentación de los contenidos a } \\
\text { enseñar a los niños/adolescentes. }\end{array}$ & 129 & 64 & 21 & 9 & 2 \\
\hline $\begin{array}{l}\text { Me sugiere estrategias para adecuar } \\
\text { los contenidos a enseñar a las } \\
\text { características de mis estudiantes y } \\
\text { los ambientes socioculturales. }\end{array}$ & 117 & 72 & 24 & 9 & 3 \\
\hline $\begin{array}{l}\text { Me ayuda a evaluar la } \\
\text { implementación de mis planes de } \\
\text { clases. }\end{array}$ & 105 & 67 & 32 & 10 & 11 \\
\hline
\end{tabular}

Fuente: elaboración propia del autor (2012)

De un total de 225 estudiantes en proceso de práctica que contestaron el instrumento, es posible observar el reconocimiento al trabajo desarrollado por el tutor en relación al ámbito Diseño del plan de clases. Específicamente, en las categorías de "bastante" a "mucho", el $86 \%$ reconoce en el tutor que les "sugiere estrategias diversas para la presentación de los contenidos a enseñar a los niños/adolescentes"; I 84\% identifica la tutoría como una instancia que les "sugiere estrategias para adecuar los contenidos a enseñar a las características de mis estudiantes y los ambientes socioculturales"; el 83\% que en relación con la comprensión del contenido a enseñar, "me ayuda a establecer vinculaciones con los objetivos transversales", el $80 \%$ considera que "retroalimenta las planificaciones clase a clase presentadas", y un $77 \%$ que les ayuda a "evaluar la implementación de mis planes de clases".

Sin embargo, se puede observar la presencia de un grupo de estudiantes que percibe en la categoría de "regular" la actuación de los tutores en este ámbito. En particular, el 13\% lo afirma en relación a la posibilidad de recibir "retroalimentación de las planificaciones clases a clase presentadas" y, congruentemente, un $14 \%$ en cuanto a "recibir ayuda para evaluar la implementación de los planes de clase".

En términos generales, los tutores estarían desarrollando una tarea clara en relación con la revisión de los diseños de aula; en facilitar la vinculación del contenido que se enseña 
con los objetivos de aprendizaje transversal (OAT); en sugerir estrategias diversas para la presentación del contenido, las cuales buscan responder a las características de los estudiantes y los contextos. No obstante, es preciso seguir avanzando en la tarea de generar las condiciones para la evaluación de aquello que previamente diseñado se termina implementando. En esa última categoría, solo el $47 \%$ de los estudiantes reconoce en sus tutores la adquisición de esta tarea. En tanto, un 18\% la considera desarrollada en términos "regulares" o "poco".

Se presenta en la tabla 3, la visión de los estudiantes en práctica respecto al funcionamiento de la tutoría, en el ámbito de la acción tutorial.

Tabla 3

Ámbito: Acción Tutorial

\begin{tabular}{|l|c|c|c|c|c|}
\hline \begin{tabular}{l} 
Ámbitos Acción Tutorial $\begin{array}{l}\text { En el trabajo de acción tutorial } \\
\text { desarrollado semanalmente, mi tutor... }\end{array}$ \\
\cline { 2 - 5 } (Mucho)
\end{tabular} & (Bastante) & (Regular) & (Poco) & (Nada) \\
\hline $\begin{array}{l}\text { Genera un espacio de trabajo } \\
\text { colaborativo en la tutoría, permitiendo la } \\
\text { conversación y el intercambio de } \\
\text { experiencias entre los estudiantes. }\end{array}$ & & 31 & 11 & 2 & 3 \\
\hline $\begin{array}{l}\text { Frente a las situaciones o eventos en los } \\
\text { que me he visto inmerso en los centros de } \\
\text { práctica, proporciona una perspectiva } \\
\text { diferente que me permite una mejor } \\
\text { comprensión de éstas. }\end{array}$ & 139 & 61 & 17 & 4 & 4 \\
\hline $\begin{array}{l}\text { Analiza posibles situaciones que puedan } \\
\text { afectar el ambiente disciplinario dentro del } \\
\text { aula. }\end{array}$ & 136 & 63 & 19 & 3 & 4 \\
\hline $\begin{array}{l}\text { Genera instancias para que me exprese y } \\
\text { dejen fluir mis emociones en función de lo } \\
\text { vivido en la práctica. }\end{array}$ & 172 & 34 & 10 & 4 & 5 \\
\hline $\begin{array}{l}\text { Elogia mis aciertos y me ayuda a corregir } \\
\text { mis equivocaciones. }\end{array}$ & 160 & 43 & 13 & 5 & 4 \\
\hline $\begin{array}{l}\text { Me entrega consejos a fin de evitar } \\
\text { decisiones académicas y/o profesionales } \\
\text { que sean apresuradas. }\end{array}$ & 149 & 62 & 8 & 2 & 4 \\
\hline $\begin{array}{l}\text { Facilita la contención emocional, el } \\
\text { detenerse y tomar distancia respecto a las } \\
\text { situaciones vividas, para analizar mejor. }\end{array}$ & 129 & 69 & 19 & 5 & 3 \\
\hline $\begin{array}{l}\text { Me ayuda a ir vinculando el conocimiento } \\
\text { teórico con la práctica, desarrollando mi } \\
\text { capacidad reflexiva. }\end{array}$ & 136 & 58 & 23 & 3 & 5 \\
\hline
\end{tabular}

Fuente: elaboración propia del autor (2012)

A la luz de los resultados, la evidencia permite constatar la presencia de una tutoría permanente concebida como espacio de aprendizaje que, desde lo personal, avanza hacia lo social y lo profesional. 
Desde lo personal, el 92\% percibe una adquisición de "mucho" y "bastante" en cuanto a que se "genera instancias para que me exprese y dejen fluir mis emociones en función de lo vivido en la práctica" y, por lo tanto, un $88 \%$ percibe la existencia de una tutoría que "facilita la contención emocional, el detenerse y tomar distancia respecto a las situaciones vividas, para analizar mejor".

Desde lo social, el 93\% percibe un importante grado de adquisición de "mucho" y "bastante" en relación con "generar un espacio de trabajo colaborativo en la tutoría, permitiendo la conversación y el intercambio de experiencias entre los estudiantes", lo que evidencia la tutoría también como instancia de socialización.

Desde lo profesional, se reconocen diversas potencialidades de la tutoría. Enb detalle, el $94 \%$ la valora, ya que "entrega consejos a fin de evitar decisiones académicas y/o profesionales que sean apresuradas"; por su parte, el $89 \%$ reconoce la potencialidad para "analizar posibles situaciones que puedan afectar el ambiente disciplinario dentro del aula"; y para el $87 \%$ "ayuda a ir vinculando el conocimiento teórico con la práctica, desarrollando mi capacidad reflexiva".

El grupo de estudiantes que se inclina en la categoría de "regular" promedia un 10\%, particularmente, el 10,2\% demanda de la tutoría la posibilidad de contribuir a establecer la integración teoría-práctica; el 7\%, espera de los tutores más ayuda para aprender a analizar las situaciones, aprendiendo a tomar distancia de las mismas, como también detenerse más en el análisis de situaciones que suelen afectar el ambiente disciplinario en el espacio del aula.

A continuación la tabla 4, presenta la visión de los estudiantes en práctica respecto a su desempeño profesional 
Tabla 4

Ámbito: Desempeño Profesional

\begin{tabular}{|c|c|c|c|c|c|}
\hline \multirow{3}{*}{$\begin{array}{l}\text { Ámbitos Desempeño profesional } \\
\text { En qué grado he desarrollado las } \\
\text { siguientes habilidades pedagógicas... }\end{array}$} & \multicolumn{5}{|c|}{ Grado de adquisición } \\
\hline & 5 & 4 & 3 & 2 & 1 \\
\hline & (Mucho) & (Bastante) & (Regular) & (Poco) & (Nada) \\
\hline $\begin{array}{l}\text { Utilizar las diversas teorías de } \\
\text { aprendizaje y desarrollo cognitivo, } \\
\text { afectivo, social y moral, para diseñar } \\
\text { diferentes situaciones de enseñanza - } \\
\text { aprendizaje. }\end{array}$ & 53 & 132 & 36 & 4 & \\
\hline $\begin{array}{l}\text { Promover el razonamiento crítico para } \\
\text { tomar decisiones de manera informada, } \\
\text { ponderando aspectos personales, } \\
\text { sociales y éticos. }\end{array}$ & 99 & 92 & 29 & 4 & 1 \\
\hline $\begin{array}{l}\text { Identificar las oportunidades que existen } \\
\text { para relacionar e integrar los } \\
\text { aprendizajes de las distintas áreas del } \\
\text { currículo, reconociendo las relaciones de } \\
\text { interdependencia que existen entre ellas. }\end{array}$ & 89 & 90 & 40 & 4 & 2 \\
\hline $\begin{array}{l}\text { Fundamentar las decisiones pedagógicas } \\
\text { que he tomado en mi planificación y } \\
\text { evaluar críticamente posibles alternativas } \\
\text { para mejorarlas, manifestando apertura } \\
\text { para recibir u ofrecer retroalimentación. }\end{array}$ & 109 & 94 & 19 & 3 & \\
\hline $\begin{array}{l}\text { Generar normas explícitas de } \\
\text { convivencia basadas en la tolerancia y } \\
\text { respeto mutuo en el aula donde } \\
\text { desarrollé mi práctica. }\end{array}$ & 155 & 57 & 11 & 2 & \\
\hline $\begin{array}{l}\text { Retroalimentar a mis estudiantes acerca } \\
\text { de sus logros académicos, con el fin de } \\
\text { estimular y desarrollar su capacidad de } \\
\text { aprendizaje y autorregulación. }\end{array}$ & 132 & 70 & 19 & 4 & \\
\hline $\begin{array}{l}\text { Comprender el rol que juega la cultura } \\
\text { escolar en el desempeño del } \\
\text { establecimiento, en el comportamiento de } \\
\text { los alumnos y en mi propia inserción } \\
\text { profesional. }\end{array}$ & 119 & 78 & 25 & 2 & 1 \\
\hline $\begin{array}{l}\text { Adaptar mi enseñanza a las } \\
\text { características de los estudiantes con } \\
\text { dificultades y con talentos especiales. }\end{array}$ & 115 & 81 & 24 & 4 & 1 \\
\hline $\begin{array}{l}\text { Identificar mis propias fortalezas y } \\
\text { debilidades como profesor en formación, } \\
\text { en relación a la disciplina que enseño, } \\
\text { reconociendo mis necesidades de } \\
\text { desarrollo profesional y actualización. }\end{array}$ & 139 & 73 & 10 & 3 & \\
\hline
\end{tabular}

Fuente: elaboración propia del autor (2012)

Los estándares señalan un "que", referido a un conjunto de aspectos o dimensiones que se debieran observar en el desempeño de un futuro profesor; y también, establecen un 
"cuanto" o medida, que permite evaluar qué tan lejos o cerca se encuentra un profesor de alcanzar un determinado desempeño.

Consultados respecto a la visión de conjunto sobre los conocimientos y habilidades profesionales, los estudiantes en práctica profesional evidencian una percepción bastante positiva del grado de adquisición de estos estándares.

Así, el 94\% reconoce haber desarrollado la habilidad pedagógica para "generar normas explícitas de convivencia basadas en la tolerancia y respeto mutuo en el aula donde desarrollé mi práctica"; un 95\% afirma haber adquirido la habilidad para "identificar mis propias fortalezas y debilidades como profesor en formación, en relación a la disciplina que enseño, reconociendo mis necesidades de desarrollo profesional y actualización"; un 91\% percibe una adquisición importante de la habilidad para "fundamentar las decisiones pedagógicas que he tomado en mi planificación y evaluar críticamente posibles alternativas para mejorarlas, manifestando apertura para recibir u ofrecer retroalimentación"; un 90\% que son capaces de "retroalimentar a mis estudiantes acerca de sus logros académicos, con el fin de estimular y desarrollar su capacidad de aprendizaje y autorregulación"; un 88\% que han evidenciado habilidad para "comprender el rol que juega la cultura escolar en el desempeño del establecimiento, en el comportamiento de los alumnos y en mi propia inserción profesional".

Sin embargo, reconocen la amplitud de tales desempeños y que, al interior de ellos, existen competencias que les reportan particular dificultad. En este sentido, identifican: "utilización de teorías del desarrollo afectivo, social y moral"; "ponderar los aspectos personales, sociales y particularmente éticos al tomar decisiones"; "integrar los aprendizajes de la distintas áreas", "mantener las normas de convivencia”, "identificar estrategias para afrontar el rol que juega la cultura escolar en la propia inserción profesional", "adaptar la enseñanza a las características de los estudiantes que más saben", y "pasar de la autoevaluación a la reflexión profunda".

Específicamente, existiría un porcentaje de estudiantes que valoran en la categoría de "regular" la adquisición de estas habilidades. De hecho, tenemos el 18\% para "identificar las oportunidades que existen para relacionar e integrar los aprendizajes de las distintas áreas del currículo, reconociendo las relaciones de interdependencia que existen entre ellas"; el $16 \%$ para el caso de "utilizar las diversas teorías de aprendizaje y desarrollo cognitivo, afectivo, social y moral, para diseñar diferentes situaciones de enseñanza aprendizaje"; el 
13\% para "promover el razonamiento crítico para tomar decisiones de manera informada, ponderando aspectos personales, sociales y éticos"; el 11\% para "comprender el rol que juega la cultura escolar en el desempeño del establecimiento, en el comportamiento de los alumnos y en mi propia inserción profesional", y el 11\% para el caso de "adaptar mi enseñanza a las características de los estudiantes con dificultades y con talentos especiales".

\section{Tutores de Práctica Profesional}

A continuación la tabla 5 presenta la visión de los tutores respecto al funcionamiento de la tutoría, en el ámbito del diseño del plan de clases.

Tabla 5

Ámbito: Diseño del Plan de Clases

\begin{tabular}{|c|c|c|c|c|c|}
\hline \multirow[t]{3}{*}{ Ámbitos Diseño del Plan de Clases } & \multicolumn{5}{|c|}{ Grado de adquisición } \\
\hline & 5 & 4 & 3 & 2 & 1 \\
\hline & (Mucho) & (Bastante) & (Regular) & (Poco) & (Nada) \\
\hline $\begin{array}{l}\text { Retroalimento las planificaciones clase a } \\
\text { clase de mis estudiantes en práctica. }\end{array}$ & 30 & 20 & 5 & 2 & \\
\hline $\begin{array}{l}\text { En relación a la comprensión del contenido a } \\
\text { enseñar, ayudo a establecer vinculaciones } \\
\text { disciplinarias e interdisciplinarias. }\end{array}$ & 28 & 26 & 3 & & \\
\hline $\begin{array}{l}\text { En relación a la comprensión del contenido a } \\
\text { enseñar, ayudo a establecer vinculaciones } \\
\text { con los objetivos transversales. }\end{array}$ & 26 & 27 & 3 & 1 & \\
\hline $\begin{array}{l}\text { Ayudo a mis estudiantes en práctica a } \\
\text { contextualizar su enseñanza en función de } \\
\text { las características de los niños/as y los } \\
\text { ambientes socioculturales. }\end{array}$ & 38 & 19 & & & \\
\hline $\begin{array}{l}\text { Ayudo a mis estudiantes en práctica a } \\
\text { evaluar la implementación de sus planes de } \\
\text { clases. }\end{array}$ & 26 & 25 & 4 & 2 & \\
\hline
\end{tabular}

Fuente: elaboración propia del autor (2012)

El tutor de práctica percibe un nivel de adquisición de "mucho" y bastante" de las tareas comprometidas en el ámbito diseño del plan de clases. En particular, "en relación a la comprensión del contenido a enseñar, ayudo a establecer vinculaciones disciplinarias e interdisciplinarias" con un 95\%, "en relación a la comprensión del contenido a enseñar, ayudo a establecer vinculaciones con los objetivos transversales", con un 93\% y "ayudo a mis estudiantes en práctica a contextualizar su enseñanza en función de las características de los niños/as y los ambientes socioculturales" con un 100\%, aparecen como las actuaciones mejor adquiridas. 
Existiría una destinación importante del tiempo de tutoría en ayudar al estudiante a favorecer diseños de aula contextualizados a las características de los estudiantes y las realidades donde intervienen. El análisis didáctico cobraría vida en este espacio formativo, cuando el practicante, a partir del dominio del conocimiento disciplinario, debe resolver tareas como la preparación de los materiales de trabajo en términos de su comprensión, progresión y vinculación con otras áreas y con objetivos de aprendizaje transversal, y las formas de presentación del contenido representado en los materiales, las estrategias de enseñanza, la respuesta a las necesidades grupales y personales de los estudiantes.

A continuación la tabla 6 presenta la visión de los tutores respecto al funcionamiento de la tutoría, en el ámbito de la acción tutorial propiamente tal.

Tabla 6

Ámbito: Acción tutorial

\begin{tabular}{|l|l|l|l|l|l|}
\hline Ámbitos Acción Tutorial \\
$\begin{array}{l}\text { En el trabajo de acción tutorial desarrollado } \\
\text { semanalmente... }\end{array}$ & \multicolumn{4}{|c|}{ Grado de adquisición } \\
\cline { 2 - 6 } & $\begin{array}{c}\text { (Mucho } \\
\text { (Bastante }\end{array}$ & (Regular) & (Poco & (Nada) \\
\hline $\begin{array}{l}\text { Favorezco un espacio de trabajo colaborativo en } \\
\text { la tutoría, permitiendo la conversación y el } \\
\text { intercambio de experiencias entre los estudiantes } \\
\text { en práctica. }\end{array}$ & & 11 & 1 & \\
\hline $\begin{array}{l}\text { Proveo de una perspectiva diferente sobre las } \\
\text { situaciones en los que el estudiante se ha visto } \\
\text { inmerso, con el fin de que tenga una mejor } \\
\text { comprensión de éstas. }\end{array}$ & & 20 & & \\
\hline $\begin{array}{l}\text { Analizo con mis estudiantes practicantes posibles } \\
\text { situaciones que puedan afectar el ambiente } \\
\text { disciplinario dentro del aula. }\end{array}$ & 36 & 17 & 3 & \\
\hline $\begin{array}{l}\text { Genero instancias para que los estudiantes se } \\
\text { expresen y dejen fluir sus emociones. }\end{array}$ & 43 & 11 & 3 & \\
\hline $\begin{array}{l}\text { Elogio a mis estudiantes practicantes respecto a } \\
\text { sus aciertos en el aula. }\end{array}$ & 47 & 9 & & \\
\hline $\begin{array}{l}\text { Entrego orientaciones a mis estudiantes en } \\
\text { práctica a fin de evitar que tomen decisiones } \\
\text { académicas y/o profesionales que sean } \\
\text { apresuradas. }\end{array}$ & 36 & 16 & 4 & \\
\hline $\begin{array}{l}\text { Favorezco la contención emocional en el } \\
\text { estudiante en práctica, el detenerse y tomar } \\
\text { distancia respecto a las situaciones vividas. }\end{array}$ & 32 & 19 & 6 & \\
\hline $\begin{array}{l}\text { Facilito la vinculación del conocimiento teórico } \\
\text { con la práctica, desarrollando en ellos la reflexión. }\end{array}$ & 34 & 20 & 2 & \\
\hline
\end{tabular}

Fuente: elaboración propia del autor (2012)

Enfrentados a la tarea de generar las condiciones para favorecer el aprender a enseñar, los tutores reconocen grados de adquisición en la categoría de "mucho": el 82\% "elogio a mis 
estudiantes practicantes respecto a sus aciertos en el aula", el $79 \%$ afirma favorecer "un espacio de trabajo colaborativo en la tutoría, permitiendo la conversación y el intercambio de experiencias entre los estudiantes en práctica" y el $76 \%$ reconoce generar "instancias para que los estudiantes se expresen y dejen fluir sus emociones".

Ahora bien, sumando las categorías "mucho" y "bastante", nos encontramos con un $100 \%$ de los tutores que reconoce proporcionar en la tutoría "de una perspectiva diferente sobre las situaciones en las que el estudiante se ha visto inmerso, con el fin de que tenga una mejor comprensión de éstas"; el 98\% que "elogio a mis estudiantes practicantes respecto a sus aciertos en el aula" y que genera "un espacio de trabajo colaborativo en la tutoría, permitiendo la conversación y el intercambio de experiencias entre los estudiantes en práctica".

En términos generales los tutores reconocen hacer de la tutoría un espacio de aprendizaje personal, social y profesional.

Finalmente, la tabla 7 presenta la visión de los tutores respecto al desempeño profesional evidenciado por los estudiantes en práctica

Tabla 7

Ámbito: Desempeño profesional

\begin{tabular}{|c|c|c|c|c|c|}
\hline \multirow{3}{*}{$\begin{array}{l}\text { Ámbitos Desempeño profesional } \\
\text { En qué grado el estudiante en práctica ha } \\
\text { desarrollado las siguientes habilidades } \\
\text { pedagógicas... }\end{array}$} & \multicolumn{5}{|c|}{ Grado de adquisición } \\
\hline & 5 & 4 & 3 & 2 & 1 \\
\hline & (Mucho) & (Bastante) & (Regular) & (Poco) & (Nada) \\
\hline $\begin{array}{l}\text { Utilizar las diversas teorías de aprendizaje y } \\
\text { desarrollo cognitivo, afectivo, social y moral, } \\
\text { para diseñar diferentes situaciones de } \\
\text { enseñanza -aprendizaje. }\end{array}$ & 3 & 15 & 29 & 9 & 1 \\
\hline $\begin{array}{l}\text { Promover el razonamiento crítico para tomar } \\
\text { decisiones de manera informada, } \\
\text { ponderando aspectos personales, sociales y } \\
\text { éticos. }\end{array}$ & 8 & 16 & 22 & 8 & 3 \\
\hline $\begin{array}{l}\text { Identificar las oportunidades que existen } \\
\text { para relacionar e integrar los aprendizajes } \\
\text { de las distintas áreas del currículo, } \\
\text { reconociendo las relaciones de } \\
\text { interdependencia que existen entre ellas. }\end{array}$ & 7 & 15 & 23 & 9 & 3 \\
\hline $\begin{array}{l}\text { Fundamentar las decisiones pedagógicas } \\
\text { que he tomado en mi planificación y evaluar } \\
\text { críticamente posibles alternativas para } \\
\text { mejorarlas, manifestando apertura para } \\
\text { recibir u ofrecer retroalimentación. }\end{array}$ & 10 & 21 & 17 & 7 & 2 \\
\hline
\end{tabular}




\begin{tabular}{|l|l|l|l|l|l|}
\hline $\begin{array}{l}\text { Generar normas explícitas de convivencia } \\
\text { basadas en la tolerancia y respeto mutuo en } \\
\text { el aula donde desarrollé mi práctica. }\end{array}$ & 18 & 22 & 12 & 3 & 2 \\
\hline $\begin{array}{l}\text { Retroalimentar a mis estudiantes acerca de } \\
\text { sus logros académicos, con el fin de } \\
\text { estimular y desarrollar su capacidad de } \\
\text { aprendizaje y autorregulación. }\end{array}$ & & 17 & 15 & 2 & 6 \\
\hline $\begin{array}{l}\text { Comprender el rol que juega la cultura } \\
\text { escolar en el desempeño del } \\
\text { establecimiento, en el comportamiento de } \\
\text { los alumnos y en mi propia inserción } \\
\text { profesional. }\end{array}$ & 25 & 12 & 7 & 3 \\
\hline $\begin{array}{l}\text { Adaptar mi enseñanza a las características } \\
\text { de los estudiantes con dificultades y con } \\
\text { talentos especiales. }\end{array}$ & 9 & 13 & 21 & 11 & 1 \\
\hline $\begin{array}{l}\text { Identificar mis propias fortalezas y } \\
\text { debilidades como profesor en formación, en } \\
\text { relación a la disciplina que enseño, } \\
\text { reconociendo mis necesidades de desarrollo } \\
\text { profesional y actualización. }\end{array}$ & & 19 & 14 & 4 & 3 \\
\hline
\end{tabular}

Fuente: elaboración propia del autor (2012)

En relación con el desempeño "utilizar las diversas teorías de aprendizaje y desarrollo cognitivo, afectivo, social y moral, para diseñar diferentes situaciones de enseñanza aprendizaje", tan solo el $31 \%$ considera que los estudiantes tienen desarrollada esta habilidad, mientras que el $50 \%$ afirma que el nivel de adquisición es "regular" y un preocupante $18 \%$ cree que los estudiantes en práctica profesional tienen "poco" o "nada" desarrollada esta actuación profesional.

En torno al desempeño "promover el razonamiento crítico para tomar decisiones de manera informada, ponderando aspectos personales, sociales y éticos", el $42 \%$ de los tutores reconoce en los practicantes una adquisición notoria; sin embargo, para el 39\% este desempeño se encuentra en un nivel de "regular" y para el 19\% este desempeño se reduce a "poco" o "nada".

En cuanto al desempeño "identificar las oportunidades que existen para relacionar e integrar los aprendizajes de las distintas áreas del currículo, reconociendo las relaciones de interdependencia que existen entre ellas" es preocupante la evaluación que realizan los tutores, pues sólo para el $38 \%$ de ellos los estudiantes en procesos de práctica logran evidenciar esta habilidad, mientras que para el $41 \%$ el desempeño se considera "regular" y para un importante $21 \%$, la adquisición se traduce en "poco" o "nada". 
Consultados respecto a "fundamentar las decisiones pedagógicas que he tomado en mi planificación y evaluar críticamente posibles alternativas para mejorarlas, manifestando apertura para recibir u ofrecer retroalimentación", el $55 \%$ de los tutores observa una adquisición notoria de esta habilidad en los practicantes. No obstante, el 30\% percibe un desarrollo "regular" de la misma en los practicantes y un $15 \%$ de los tutores prácticamente no han adquirido la habilidad para evaluar y tomar decisiones fundamentadas a las problemáticas que enfrenta.

Un panorama distinto se observa en el desempeño "generar normas explícitas de convivencia basadas en la tolerancia y respeto mutuo en el aula donde desarrollé mi práctica", pues el 71\% afirma una adquisición de "bastante" y "mucho" en los desempeños asociados a normas de convivencia. Sin embargo, el $21 \%$ percibe un desempeño "regular" en esta dimensión y el 8\% lo observa como una habilidad que los estudiantes no estarían evidenciando.

En lo referido a "retroalimentar a mis estudiantes acerca de sus logros académicos, con el fin de estimular y desarrollar su capacidad de aprendizaje y autorregulación", el 50\% de los tutores lo percibe como una habilidad evidenciada por los estudiantes en sus procesos de práctica; en tanto, el $26 \%$ lo observa con un desempeño "regular" en sus practicantes y para el $14 \%$ existirían serías falencias en los mismos, pues su nivel de adquisición se reduce a "poco" y "nada".

En cuanto a "comprender el rol que juega la cultura escolar en el desempeño del establecimiento, en el comportamiento de los alumnos y en mi propia inserción profesional" el $62 \%$ de los tutores observa una adquisición de "mucho" y "bastante" en sus practicantes. No obstante, el $21 \%$ lo califica de "regular" y un $17 \%$ estima que la adquisición de esta habilidad se reduce a "poco" y "nada".

Respecto a "adaptar la enseñanza a las características de los estudiantes con dificultades y con talentos especiales", nos encontramos con falencias importantes. El 37\% considera que la adquisición de esta habilidad se encuentra en la categoría de "regular" y para el $24 \%$ de los tutores, esta habilidad no está adquirida en los estudiantes. Solo un 39\% considera que sus practicantes evidencian una adquisición de "mucha" o "bastante" en relación con ser capaz de contextualizar la enseñanza, respondiendo a los requerimientos de los estudiantes y los contextos de los cuales provienen. 
Finalmente, en lo referido a "identificar las propias fortalezas y debilidades como profesor en formación, en relación con la disciplina que enseño, reconociendo mis necesidades de desarrollo profesional", el 63\% reconoce un nivel de adquisición de "mucho" y "bastante" a la hora de autoevaluar el propio desempeño. Sin embargo, existiría un 25\% que observa dificultades en el practicante para autoevaluarse en función de su quehacer y un 12\% considera que, definitivamente, esta habilidad no es evidente en el desempeño del estudiante que enfrenta su proceso de práctica profesional.

Por lo tanto, en términos genéricos las habilidades mejor adquiridas por los practicantes a partir de la evidencia aportada por los tutores sería "generar normas explícitas de convivencia basadas en la tolerancia y respeto mutuo en el aula donde desarrolla la práctica" y "comprender el rol que juega la cultura escolar en el desempeño del establecimiento, en el comportamiento de los alumnos y en la propia inserción profesional". Curiosamente una competencia muy radicada en el espacio del aula, la primera y, anclada en lo institucional, la segunda. Por su lado, emergen como habilidades necesarias de fortalecer "utilizar las diversas teorías de aprendizaje y desarrollo cognitivo, afectivo, social y oral, para diseñar diferentes situaciones de enseñanza-aprendizaje", "identificar las oportunidades que existen para relacionar e integrar los aprendizajes de las distintas áreas del currículo, reconociendo las relaciones de interdependencia que existen entre ellas" y "adaptar mi enseñanza a las características de los estudiantes con dificultades y con talentos especiales". Estas tres últimas bastante relacionadas con el espacio natural del trabajo docente como es el aula y el proceso de enseñanza -aprendizaje.

\section{Conclusiones}

La práctica en general constituye el espacio donde deben confluir la teoría (conocimientos y creencias) y la realidad (y sus diversos contextos y exigencias) y cuya relación es enfrentada por un estudiante en formación, y resuelta favorablemente en la medida en que es capaz de abandonar certezas a fin de exponerse a la docencia, evidenciando ciertos desempeños.

Desde otra perspectiva, un momento de particular complejidad entre lo que podría ser "la alegre teoría en las nubes" y la dura práctica cotidiana, de no mediar un sistema de acompañamiento que permita que estos inicios conflictivos puedan ser "suavizados" en la 
medida en que generemos oportunidades para comprender la ecología de los contextos escolares, como bien lo plantea (Orland-Barak, 2008).

Es así como nos encontramos con opiniones bastante positivas respecto al sistema de acompañamiento diseñado por la universidad para los estudiantes en práctica profesional y que se concretan en la presencia de un profesional encargado de apoyar esta etapa de la formación. De este modo, practicantes como tutores de práctica reconocen un trabajo que permite apoyar las exigencias personales, sociales y profesionales que experimentan los practicantes en lo referido a la contención emocional, el detenerse y tomar distancia respecto a las situaciones que se viven para desarrollar miradas más analíticas, permitir la expresión y emociones del estudiante en función de lo que está enfrentando y viviendo en sus centros de práctica; generar espacios de colaboración, posibilitando la conversación y el intercambio de experiencias entre estudiantes y, sin duda, aportar nuevas perspectivas para una mejor comprensión de las situaciones o eventos donde los practicantes se ven inmersos y con ello ayudar en la vinculación del conocimiento teórico con la práctica, desarrollando la capacidad reflexiva.

En relación con los referentes teóricos presentados, la tutoría estaría permitiendo "suavizar" la complejidad de este momento del itinerario formativo (Galaz, 2011, p.49); con un tutor que preferentemente desarrollaría funciones vinculadas a la de apoyo, de aculturador y educador, en tanto es un sujeto que siempre "está presente", proporcionando oportunidades para que los practicantes se expresen y dejen fluir sus emociones, actuando como caja de resonancia ante situaciones que implica la docencia; articulando ideas y creando conscientemente los andamiajes apropiados de aprendizaje profesional para el estudiante en práctica, como es lo esperado según Malderez (2004).

Desde un punto de vista más técnico o profesional, se valora la retroalimentación de las clases planificadas y ejecutadas; la sugerencia de diversas estrategias para la presentación de los contenidos a enseñar; la adecuación de los mismos en función de las características de los estudiantes y los ambientes socioculturales; la vinculación que se logra con los objetivos transversales, tareas que, sin duda, suponen una intensa exigencia a los tutores de práctica.

Lo sorprendente es que las opiniones de estudiantes en práctica y tutores muestran bastante similitud, y podrían llevarnos a sostener que estos espacios formativos están efectivamente instalados, con sesiones de funcionamiento semanal de información y organización del trabajo, tal cual lo contempla la propuesta de la facultad de educación. 
Siguiendo con las conclusiones, nos interesó aproximarnos a los desempeños profesionales evidenciados por los practicantes en sus diversos contextos y, en ese sentido, emergen algunos elementos que generan más de una sorpresa. Llama la atención que si bien se reconoce la tutoría como el espacio de apoyo al desempeño del practicante en aula en lo referido a la planificación, la didáctica, la evaluación y la reflexión, la visión que existe de los desempeños logrados tiende a ser distinta entre los actores.

Los practicantes perciben un desempeño que, en términos generales, se sitúa en los niveles de "bastante" y "mucho", lo cual indicaría que, desde su mirada, consideran adquiridos los distintos desempeños pedagógicos; no obstante, la óptica de los tutores es diferente, y sitúan a un porcentaje importante de practicantes en una adquisición más bien parcial o "regular" de los desempeños pedagógicos comprometidos en la formación de un profesor. Lo curioso es que, por una parte, se reconoce el fuerte apoyo técnico entregado al practicante en el contexto de tutoría, pero pareciera que estos apoyos no estarían siendo suficientes en relación a los desempeños finalmente movilizados en los contextos de práctica. $\mathrm{O}$ que los tutores no estarían en condiciones de revertir ciertos aspectos no suficientemente desarrollados durante el proceso formativo de un estudiante de pedagogía. O que la misma ecología de los contextos donde se desarrollan las prácticas, y que refieren a las características de los sujetos y los diversos escenarios, estarían generando demandas que la formación inicial no logra atender. Estas diversas lecturas, abren oportunidades para revisar el tipo de vinculación entre la tutoría y el proyecto formativo en su conjunto y, en consecuencia, retroalimentarlo con la evidencia recogida.

Lo anterior pone de manifiesto la complejidad que encierra la docencia y la exigencia que la formación tiene respecto a generar las condiciones, los escenarios y las interacciones que permitan a los futuros docentes aprender a pensar, a hablar y a actuar como profesores. Solo de esa manera será posible deslizar el velo de sombra que parece rodear los procesos de formación inicial en relación con las competencias necesarias para el adecuado desarrollo del proceso de enseñanza, independiente de la disciplina que se enseñe. Esto cobra particular relevancia si se considera que la formación inicial explica, o debería explicar, la calidad del quehacer de un profesor, formación que, como hemos advertido, se pone a prueba en los procesos de práctica profesional y se traduce en un conjunto de desempeños.

Al respecto, muchas interrogantes emergen, por ejemplo: ¿será que los modelos teóricos basados en la filosofía profesada no están coincidiendo con su teoría al uso?; 
¿tenemos verdadera conciencia de los desafíos que encierran los distintos desempeños?; ¿cómo los estamos comprendiendo y aplicando?; ¿con qué rigor los estamos evaluando?; ¿se están correspondiendo estas valoraciones de los desempeños de los practicantes con los niveles de aprobación y reprobación que finalmente se evidencia en esta etapa de la formación?; ¿la visión positiva de los desempeños por parte de nuestros estudiantes se corresponde con la capacidad reflexiva que comprometen todos los proyectos de carrera?

En consecuencia, estas interrogantes constituyen una fuente de oportunidad, pues invitan a generar espacios de discusión académica que permitan asegurar, entre quienes participan de los procesos de formación de profesores, el conocimiento, la comprensión y la apropiación de los desempeños pedagógicos esperables en nuestros estudiantes. A partir de ahí, surgen una serie de interrogantes con potencial investigativo, tales como ¿qué y cómo los practicantes están utilizando las diversas teorías de aprendizaje y desarrollo cognitivo, afectivo, social y moral para el diseño de situaciones pedagógicas?, ¿logran ponderar los aspectos personales, sociales y éticos en la toma de decisiones de las situaciones que enfrentan?, ¿pueden reconocer las relaciones de interdependencia entre aprendizajes de distintas áreas?, ¿están siendo capaces de fundamentar sus decisiones pedagógicas?, ¿les resulta posible retroalimentar a los estudiantes de sus aulas para ayudarles a la autorregulación?, ¿logran comprender el rol que juega la cultura escolar en el desempeño del establecimiento, en el comportamiento de los alumnos y en su propia inserción profesional?, ¿están evidenciando adaptación de la enseñanza a las características de los estudiantes con dificultades y con talentos específicos?, etc. preguntas que aluden al conjunto de desempeños que, en su conjunto, deberían estar en la base de lo que se declara y termina haciendo efectivo en la línea de formación práctica, y en los distintos proyectos de carrera, pues aluden a los conocimientos, habilidades y actitudes esperables del egresado de pedagogía, que deberían estar encontrando expresión en los rediseños curriculares en proceso.

\section{Recomendaciones}

- Develar modelos y enfoques que subyacen a la práctica profesional de las distintas carreras, alineando los procesos (estructura y contextos) teniendo en consideración los Estándares Orientadores para Egresados y el Marco para la Buena Enseñanza y las demandas derivadas de la ecología de los contextos escolares. 
- Definición de instrumentos que permitan hacer más explícita, operacional y, por lo tanto, evaluables las dimensiones asociadas a cada uno de los desempeños pedagógicos y disciplinarios comprometidos en la tarea docente.

- Coordinación de los equipos que participan en prácticas con el propósito de asegurar un nivel de conocimiento compartido del conjunto del proyecto formativo de la Facultad; de los compromisos - de conocimientos, habilidades y actitudes- que representan los distintos desempeños pedagógicos; la naturaleza de las actividades de tutoría y apoyo; la coherencia entre nuestra actuación didáctica y su evaluación; la variedad y gradualidad de las demandas -de conocimiento o habilidad- que les planteamos a los estudiantes; la importancia de los niveles de información previa y de feedback posterior suministrado a los estudiantes para que puedan mejorar, y los niveles de exigencia y rigor con que finalmente se evalúa el desempeño.

\section{Referencias}

Álvarez Pedro. (2002). La función tutorial en la Universidad. Una apuesta en la mejora de la calidad de enseñanza. Madrid: EOS Universitaria.

Ball, Deborah and Cohen, David. (1999). Developing Practice, Developing Practitioners. Toward a Practice- Based Theory of Professional Education. In Linda Darling-Hammond y Gary Sykes (Eds.), Teaching as a Learning Profession: handbook of policy and practice (pp. 3-32). San Francisco: Jossey-Bass.

Brockbank Anne y McGill, Ian. (2002). Aprendizaje reflexivo en la educación superior. Madrid: Ediciones Morata.

Galaz Alberto (ed.). (2011). Estrategias reflexivas en la formación de profesores y de formadores de profesores. Santiago de Chile: LOM.

Malderez, Angi y Bodóczky C. (2004). Mentor Courses: A resource book for trainer-trainer. Londres:Cambridge University Press, United Kingdom.

Marcelo, G., Carlos (2008). Profesores Principiantes e inserción a la docencia. Barcelona: Ediciones Octaedro.

Martin, Susan D., Snow, Jennifer L., y Franklin, Cheryl A. (2011). Navegating the terrain of third space: Tension with/in relationships in school - university partnerships. Journal of Teacher Education, 62(39), 299-311.

Montecinos Carmen, Solís, María Cristina, Contreras, Inés y Rittershaussen, Sylvia. (2009). Muestras de desempeño docente. Santiago de Chile: Ediciones UC. 
Orland-Barak, Lily y Yinon, H. (2008). El impacto de las experiencias prácticas en los profesores principiantes: mentorazgo para formular preguntas diferentes. En Carlos Marcelo (Ed.), Profesores principiantes e inserción a la docencia (pp. 155-176). Barcelona. Octaedro.

Pérez Gómez, Augusto. (1988). El pensamiento práctico del profesor: Implicaciones en la Formación del Profesorado. En Aurelio Villa (coord.), Perspectivas y problemas de la función docente (pp. 128-148). Madrid: Octaedro.

Universidad Autónoma de Chile. (2012). Proyecto de carrera. Temuco, Chile: Facultad de Educación, UAC. 